PROCEEDINGS OF THE

AMERICAN MATHEMATICAL SOCIETY

Volume 127, Number 3, March 1999, Pages 763-769

S 0002-9939(99)04750-4

\title{
A NECESSARY CONDITION OF SOLVABILITY FOR THE CAPILLARITY BOUNDARY OF MONGE-AMPERE EQUATIONS IN TWO DIMENSIONS
}

\author{
MA XI-NAN \\ (Communicated by Peter Li)
}

\begin{abstract}
In this paper we consider a class of Monge-Ampere equations with a prescribed contact angle boundary value problem on a bounded strictly convex domain in two dimensions. The purpose is to give a sharp necessary condition of solvability for the above mentioned equations. This is achieved by using the maximum principle and introducing a curvilinear coordinate system for Monge-Ampere equations in two dimensions. An interesting feature of our necessary condition is the need for a certain strong restriction between the curvature of the boundary of domain and the boundary condition, which does not appear in the Dirichlet and Neumann boundary values.
\end{abstract}

\section{$\S 1$. INTRODUCTION}

The existence of convex classical solutions of the Dirichlet boundary problem for equations of Monge-Ampere type,

$$
\begin{aligned}
\operatorname{det} u_{i j} & =f(x, u, D u) \quad \text { in } \quad \Omega, \\
u & =\phi(x) \quad \text { on } \quad \partial \Omega,
\end{aligned}
$$

where $\Omega$ is a convex domain in $R^{n}, f$ is a prescribed positive function on $\Omega \times R \times R^{n}$, $\phi(x) \in C^{\infty}(\bar{\Omega})$, have been obtained in [1]. In conjunction with (1.1), the Neumannn boundary and the oblique derivative problem also have been considered in [2], [5], [6], [7]. They established various existence theorems. For the nonlinear oblique value problems, the only known result is obtained in the paper [7] of Urbas. But his result excludes the following capillarity boundary problem:

$$
\frac{\partial u}{\partial n}=\cos \theta(x, u) \sqrt{1+|D u|^{2}} \quad \text { on } \quad \partial \Omega,
$$

where $n$ is the unit normal vector pointing outward from $\partial \Omega$ and $\theta(x, u) \in(0, \pi)$ is the wetting angle. The reason for excluding the above case is that the second derivative estimates may fail to hold. So even for the equations

$$
\begin{aligned}
\operatorname{det} u_{i j} & =c & & \text { in } \quad \Omega, \\
\frac{\partial u}{\partial n} & =\cos \theta_{o} \sqrt{1+|D u|^{2}} & & \text { on } \quad \partial \Omega,
\end{aligned}
$$

Received by the editors June 16, 1997.

1991 Mathematics Subject Classification. Primary 35J25, 35J60, 35J65; Secondary 53C45.

Key words and phrases. Monge-Ampere equation, maximum principle, curvilinear coordinate system, contact angle boundary. 
where $\Omega$ is a strictly convex bounded smooth domain in $R^{2}, c$ is a positive constant, and $\theta_{o} \in\left(0, \frac{\pi}{2}\right)$, the existence of a classical solution is still open.

In this paper we give a necessary condition of solvability for (1.4)-(1.5); more precisely we obtain the following theorem.

Theorem. Under the above hypotheses on $\Omega, c, \theta_{o}$, if $u(x) \in C^{2}(\bar{\Omega}) \cap C^{3}(\Omega)$ is the strictly convex solution of (1.4)-(1.5), then we have the following relation

$$
k_{0} \leq \max \left\{\sqrt{c} \cos \theta_{o}, \sqrt{c} \tan \theta_{o}\right\},
$$

where $k_{0}=\min _{x \in \partial \Omega} k(x)>0$ and $k(x)$ is the curvature of $\partial \Omega$ at $x$.

The above theorem shows that when we solve capillarity boundary value problems for Monge-Ampere equations on a strictly convex domain in $R^{2}$, there exists a strong restriction between the curvature of the boundary of domain and the boundary value, which is different from Dirichlet and Neumann boundary value problems $[1],[2]$.

Remark 1.1. When $\theta_{o}=\frac{\pi}{5}, \cos \theta_{o}=\tan \theta_{o}=\frac{\sqrt{5}-1}{2}$, and there exists a point $x_{o} \in \partial \Omega$ such that $k\left(x_{o}\right) \leq \frac{\sqrt{5}-1}{2}$, then our condition (1.6) is automatically satisfied.

The proof of the theorem depends on the maximum principle for some suitable auxillary function and the introduction of a curvilinear coordinate system for Monge-Ampere equations in two dimensions. In section 2, we will state some notations and lemmas. The proof of the theorem and an example which implies the sharpness of our condition will be given in section 3. In [3], the same technique will also be used to obtain the isoperimetric bounds of classical convex solutions of homogeneous Dirichlet and Robin boundary values for the Monge-Ampere equation

$$
\operatorname{det} u_{i j}=c,
$$

in two dimensions.

\section{$\S 2$. Notations and Lemmas}

Let $C$ be a curve in the plane given in parametric representation by

$$
x^{i}=x^{i}(s), \quad i=1,2,
$$

where $s$ denotes the arc length along $C$ measured from some point on $C$. Then for the tangent vector $T$, we have $T^{i}=\dot{x}^{i},|T|^{2}=1, \dot{x}^{i}(s)=\frac{d x^{i}(s)}{d s}$.

Assume now that a function $u(x)$ is defined on the closure of a bounded convex smooth domain $\Omega$ with $\partial \Omega$ as its boundary; we denote $n$ as its unit outward normal. As in [4], let's introduce the curvilinear coordinate system $(s, r)$ with $\partial \Omega$ as the reference curve; here $s$ stands for the arc-length of $\partial \Omega$ and $r$ is taken to be positive in the direction of $n$. Then define the "normal derivative" $\frac{\partial u}{\partial n}$ of $u$ by

$$
\frac{\partial u}{\partial n}=\lim _{r \rightarrow 0} \frac{1}{r}\left(u\left(x^{i}\right)-u\left(x^{i}-r n^{i}\right)\right) .
$$

Meanwhile we have

$$
u\left(x^{1}, x^{2}\right)=u\left(x^{1}(s), x^{2}(s)\right)=u(s) \quad \text { on } \quad \partial \Omega,
$$


so we can also define a tangential derivative by

$$
\frac{\partial u}{\partial s}=\frac{d u}{d s}=\frac{\partial u}{\partial x^{i}} \dot{x}^{i}=u_{i} T^{i}
$$

We also denote

$$
u_{i}=\frac{\partial u}{\partial x_{i}}, \quad u_{i j}=\frac{\partial^{2} u}{\partial x_{i} \partial x_{j}} .
$$

Then we have the following formulas at any point $x \in \partial \Omega$ :

$$
\begin{aligned}
\frac{\partial u}{\partial s} & =u_{i} T^{i}, \\
\frac{\partial u}{\partial n} & =u_{i} n^{i}, \\
\frac{\partial^{2} u}{\partial^{2} s} & =u_{i j} T^{i} T^{j}-k u_{i} n^{i}, \\
\frac{\partial^{2} u}{\partial^{2} n} & =u_{i j} n^{i} n^{j}, \\
\frac{\partial}{\partial s}\left(\frac{\partial u}{\partial n}\right) & =u_{i j} n^{i} T^{j}+k u_{i} T^{i}, \\
\frac{\partial}{\partial n}\left(\frac{\partial u}{\partial s}\right) & =\frac{\partial}{\partial s}\left(\frac{\partial u}{\partial n}\right)-k \frac{\partial u}{\partial s}=u_{i j} n^{i} T^{j} .
\end{aligned}
$$

Lemma 2.1. For $u \in C^{2}(\bar{\Omega})$, we have the following relation at any point $x_{0} \in \partial \Omega$ :

$$
u_{i j}=\sum_{k, l=1}^{2}\left(T^{i} T^{j} T^{k} T^{l}+T^{i} n^{j} T^{k} n^{l}+n^{i} T^{j} n^{k} T^{l}+n^{i} n^{j} n^{k} n^{l}\right) D_{k l} u .
$$

Proof. This can be verified directly by using

$$
T^{1}=n^{2}, \quad T^{2}=-n^{1} .
$$

Lemma 2.2. For $u \in C^{2}(\bar{\Omega})$, the equation $\operatorname{det} u_{i j}=c$ can be rewritten on $\partial \Omega$ in the following form:

$$
\left(\frac{\partial^{2} u}{\partial s^{2}}+k \frac{\partial u}{\partial n}\right) \frac{\partial^{2} u}{\partial n^{2}}=c+\left[\frac{\partial}{\partial n}\left(\frac{\partial u}{\partial s}\right)\right]^{2} .
$$

Proof. This follows from Lemma 2.1 and the formulas (2.2)-(2.7).

Lemma 2.3. Suppose that $P(x)=|D u|^{2}-2 \sqrt{c} u$, where $u \in C^{2}(\bar{\Omega}) \cap C^{3}(\Omega)$ is a strictly convex solution of equation (1.4). Then $P(x)$ attains its maximum on $\partial \Omega$.

Proof. Without loss of generality, assume that $P(x)$ is not a constant in $\bar{\Omega}$. By using the summation convention, we have

$$
\begin{aligned}
P_{i} & =2 u_{i} u_{i j}-2 \sqrt{c} u_{i}, \\
P_{i j} & =2 u_{k j} u_{k i}+2 u_{k} u_{k i j}-2 \sqrt{c} u_{i j} .
\end{aligned}
$$

From equation (1.4), it follows that

$$
\log \left(\operatorname{det} u_{i j}\right)=\log c .
$$

Differentiating (2.12) with respect to $x_{k}$, we have

$$
u^{i j} u_{i j k}=0, \quad k=1,2,
$$


where $\left\{u^{i j}\right\}$ is the inverse of the Hessian matrix $H=\left\{u_{i j}\right\}$. Since $u \in C^{2}(\bar{\Omega})$ is strictly convex, $\left\{u^{i j}\right\}$ is a positive definite matrix, and therefore

$$
\begin{aligned}
u^{i j} P_{i j} & =2 u^{i j} u_{k j} u_{k i}+2 u^{i j} u_{k} u_{k i j}-2 \sqrt{c} u^{i j} u_{i j} \\
& =2 \Delta u-4 \sqrt{c} .
\end{aligned}
$$

Now use (1.4) to obtain

$$
\Delta u=u_{11}+u_{22} \geq 2 \sqrt{u_{11} u_{22}}=2 \sqrt{c+u_{12}{ }^{2}} \geq 2 \sqrt{c} .
$$

So

$$
u^{i j} P_{i j} \geq 0
$$

and then $P(x)$ attains its maximum on $\partial \Omega$ by the maximum principle.

\section{§3. The Proof of the Theorem}

In this section the Theorem will be proved by a careful analysis of $P(x)$ on $\partial \Omega$.

Proof. If $u \in C^{2}(\bar{\Omega}) \cap C^{3}(\Omega)$ is a strictly convex solution to the equations (1.4)(1.5), by Lemma 2.3, $P(x)$ takes its maximum at some point $x_{o} \in \partial \Omega$. From the Hopf boundary point lemma [4], we have either

$$
P(x) \equiv \text { Const. } \quad \text { in } \bar{\Omega},
$$

or

$$
\frac{\partial P}{\partial n}\left(x_{o}\right)>0
$$

(i): Suppose first that $P(x)$ is not a constant. We then have at $x_{o} \in \partial \Omega$,

$$
\begin{aligned}
& \frac{\partial P}{\partial s}=0, \\
& \frac{\partial P}{\partial n}>0 .
\end{aligned}
$$

Now, (3.1) and (3.2) may be written explicity at $x_{o}$ as follows:

$$
\begin{aligned}
& \frac{\partial P}{\partial s}=2 \frac{\partial u}{\partial s} \cdot \frac{\partial}{\partial s}\left(\frac{\partial u}{\partial s}\right)+2 \frac{\partial u}{\partial n} \cdot \frac{\partial}{\partial s}\left(\frac{\partial u}{\partial n}\right)-2 \sqrt{c} \frac{\partial u}{\partial s}=0, \\
& \frac{\partial P}{\partial n}=2 \frac{\partial u}{\partial n} \cdot \frac{\partial}{\partial n}\left(\frac{\partial u}{\partial n}\right)+2 \frac{\partial u}{\partial s} \cdot \frac{\partial}{\partial n}\left(\frac{\partial u}{\partial s}\right)-2 \sqrt{c} \frac{\partial u}{\partial n}>0 .
\end{aligned}
$$

From the boundary condition (1.5), we get

$$
\frac{\partial u}{\partial n} \cdot \frac{\partial}{\partial s}\left(\frac{\partial u}{\partial n}\right)=\cot ^{2} \theta_{o} \cdot \frac{\partial u}{\partial s} \frac{\partial}{\partial s}\left(\frac{\partial u}{\partial s}\right) .
$$

Inserting into (3.3) yields

$$
\frac{\partial P}{\partial s}=2 \frac{\partial u}{\partial s}\left(\frac{1}{\sin ^{2} \theta_{o}} \cdot \frac{\partial^{2} u}{\partial s^{2}}-\sqrt{c}\right)=0 \quad \text { at } \quad x_{o} .
$$

Thus, the following two cases will occur.

Case (a):

$$
\frac{\partial u}{\partial s}\left(x_{o}\right)=0 .
$$


In this case, we may use the fact that $\frac{\partial^{2} P}{\partial s^{2}}\left(x_{o}\right) \leq 0$. But since $\frac{\partial u}{\partial s}\left(x_{o}\right)=0$,

$$
\frac{\partial^{2} P}{\partial s^{2}}=2 \frac{\partial^{2} u}{\partial s^{2}}\left(\frac{1}{\sin ^{2} \theta_{o}} \cdot \frac{\partial^{2} u}{\partial s^{2}}-\sqrt{c}\right) \leq 0 \quad \text { at } \quad x_{o}
$$

i.e. at $x_{o}$ we have

$$
0 \leq \frac{\partial^{2} u}{\partial s^{2}} \leq \sqrt{c} \sin ^{2} \theta_{o}
$$

From (3.3) and (3.7) we have

$$
\frac{\partial}{\partial s}\left(\frac{\partial u}{\partial n}\right)\left(x_{o}\right)=0
$$

So by $(2.7)$

$$
\frac{\partial}{\partial n}\left(\frac{\partial u}{\partial s}\right)\left(x_{o}\right)=0
$$

Therefore (2.9) can be written as

$$
\frac{\partial^{2} u}{\partial n^{2}}=\frac{c}{\frac{\partial^{2} u}{\partial s^{2}}+k \frac{\partial u}{\partial n}} \quad \text { at } x_{o}
$$

Inserting into (3.4) yields

$$
\frac{\partial P}{\partial n}=2 \frac{\partial u}{\partial n} \frac{c}{\frac{\partial^{2} u}{\partial s^{2}}+k \frac{\partial u}{\partial n}}-2 \sqrt{c} \frac{\partial u}{\partial n}>0 \quad \text { at } x_{o} .
$$

From $\frac{\partial u}{\partial s}\left(x_{o}\right)=0$ and the boundary value condition, we have

$$
\frac{\partial u}{\partial n}\left(x_{o}\right)=\cot \theta_{o}
$$

From (3.9), (3.13) and (3.14), we have that

$$
k\left(x_{o}\right) \leq \sqrt{c} \tan \theta_{o} .
$$

Case (b):

$$
\frac{\partial u}{\partial s}\left(x_{o}\right) \neq 0 .
$$

Then it follows from (3.6) that we have

$$
\frac{\partial^{2} u}{\partial s^{2}}=\sqrt{c} \sin ^{2} \theta_{o} \quad \text { at } x_{o} .
$$

Combining (3.5), (3.17) and (2.7), we have

$$
\frac{\partial}{\partial n}\left(\frac{\partial u}{\partial s}\right)=\frac{\partial u}{\partial s}\left(\frac{\sqrt{c} \cos ^{2} \theta_{o}}{\frac{\partial u}{\partial n}}-k\right) \quad \text { at } x_{o} .
$$

If we insert the expression (3.18) into (2.9), we get

$$
\frac{\partial^{2} u}{\partial n^{2}}=\frac{c+\left(\frac{\partial u}{\partial s}\right)^{2}\left[\frac{\sqrt{c} \cos ^{2} \theta_{o}}{\frac{\partial u}{\partial n}}-k\right]^{2}}{\sqrt{c} \sin ^{2} \theta_{o}+k \frac{\partial u}{\partial n}} \text { at } x_{o} .
$$


In this case the expression (3.4) becomes

$$
k \frac{\partial u}{\partial n} \leq \sqrt{c} \cos ^{2} \theta_{o} \quad \text { at } \quad x_{o} .
$$

Thus from the boundary value condition (1.5), we have

$$
k\left(x_{o}\right) \leq \sqrt{c} \cos \theta_{o} .
$$

(ii): If $P(x) \equiv$ Const. on $\bar{\Omega}$, then for any point $x_{o} \in \partial \Omega$, we have

$$
\begin{aligned}
& \frac{\partial P}{\partial s}=2 \frac{\partial u}{\partial s} \cdot \frac{\partial}{\partial s}\left(\frac{\partial u}{\partial s}\right)+2 \frac{\partial u}{\partial n} \cdot \frac{\partial}{\partial s}\left(\frac{\partial u}{\partial n}\right)-2 \sqrt{c} \frac{\partial u}{\partial s}=0 \\
& \frac{\partial P}{\partial n}=2 \frac{\partial u}{\partial n} \cdot \frac{\partial}{\partial n}\left(\frac{\partial u}{\partial n}\right)+2 \frac{\partial u}{\partial s} \cdot \frac{\partial}{\partial n}\left(\frac{\partial u}{\partial s}\right)-2 \sqrt{c} \frac{\partial u}{\partial n}=0 .
\end{aligned}
$$

If $\frac{\partial u}{\partial s}\left(x_{o}\right)=0$, then at $x_{o}$

$$
\frac{\partial^{2} P}{\partial s^{2}}=2 \frac{\partial^{2} u}{\partial s^{2}}\left(\frac{1}{\sin ^{2} \theta_{o}} \frac{\partial^{2} u}{\partial s^{2}}-\sqrt{c}\right)=0 .
$$

So it may be may divided into three cases.

Case (c):

$$
\frac{\partial u}{\partial s}\left(x_{o}\right)=0, \quad \frac{\partial^{2} u}{\partial s^{2}}\left(x_{o}\right)=\sqrt{c} \sin ^{2} \theta_{o} .
$$

As in case (a), (3.22)-(3.23) and (3.25) imply

$$
k \frac{\partial u}{\partial n}=\sqrt{c} \cos ^{2} \theta_{o} \quad \text { at } x_{o} .
$$

So from (3.14), it follows that

$$
k\left(x_{o}\right)=\sqrt{c} \sin \theta_{o} \cos \theta_{o} .
$$

Case (d):

$$
\frac{\partial u}{\partial s}\left(x_{o}\right)=0, \quad \frac{\partial^{2} u}{\partial s^{2}}\left(x_{o}\right)=0 .
$$

Again as in case (a), (3.22)-(3.23) and (3.28) imply

$$
k\left(x_{o}\right)=\sqrt{c} \tan \theta_{o} .
$$

Case (e):

$$
\frac{\partial u}{\partial s}\left(x_{o}\right) \neq 0, \quad \frac{\partial^{2} u}{\partial s^{2}}\left(x_{o}\right)=\sqrt{c} \sin ^{2} \theta_{o} .
$$

Then as in case (b), by (3.22)-(3.23) and (3.30) we have

$$
k\left(x_{o}\right) \leq \sqrt{c} \cos \theta_{o} .
$$

So from (3.15), (3.21), (3.27), (3.29) and (3.31), we know that if $u \in C^{2}(\bar{\Omega}) \cap$ $C^{3}(\Omega)$ is the strictly convex solution for equations (1.4)-(1.5), then the following inequality holds:

$$
k_{o}=\min _{x \in \partial \Omega} k(x) \leq \max \left\{\sqrt{c} \cos \theta_{o}, \quad \sqrt{c} \tan \theta_{o}\right\},
$$

which completes the proof of the theorem.

Now we give an example that shows the upper bound of $k(x)$ is sharp. 
Example. When $\Omega=B(o, R)$, the disk with radius $R$ in $R^{2}$, and one considers $u=x^{2}+y^{2}$, then $\operatorname{det}\left(u_{i j}\right)=4$ and

$$
\frac{\partial u}{\partial n}=2 R, \quad 1+|D u|^{2}=1+4 R^{2}, \quad \text { on } \quad \partial \Omega .
$$

Thus, the boundary condition

$$
4 R^{2}=\cos ^{2} \theta_{o}\left(1+4 R^{2}\right)
$$

implies

$$
4 R^{2}=\cot ^{2} \theta_{o}
$$

and

$$
k(x)=\frac{1}{R}=2 \tan \theta_{o} .
$$

This examples shows that for some $\theta_{o}$ with $\tan \theta_{o} \geq \cos \theta_{o}$, the condition (1.6) is sharp.

\section{ACKNOWLEDGEMENTS}

The author thanks the referee for suggesting the above example.

\section{REFERENCES}

1. L.Caffarelli, L.Nirenberg and J.Spruck, The Dirichlet Problem for Nonlinear Second-Order Elliptic Equations I.Monge-Ampere Equation, Comm. Pure App. Math. 37 (1984), 369-402. MR 88k:35073

2. P.L.Lions, N.S.Trudinger and J.Urbas, The Neumann problem for equations of Monge-Ampere type, Comm. Pure Appl. Math. 39 (1986), 539-563. MR 87j:35114

3. Ma Xi-nan, Isoperimetric bounds for Monge-Ampere equations in two dimensions, to appear in Analysis.

4. R.P.Sperb, Maximum Principles and Their Applications, Academic Press,New York, 1981. MR 84a:35033

5. N.S.Trudinger, On degenerate fully nonlinear ellptic equations in balls, Bull. Aust. Math. Soc. 111 (1987), 299-307. MR 88b:35083

6. J.Urbas, The oblique derivative problem for equations of Monge-Ampere type, Proceeding of the Centre for Mathematical Analysis, Australian National University 12 (1987), 171-195. MR 89b:35045

7. J.Urbas, Nonlinear oblique boundary value problem for Hessian equations in two dimensions, Ann. Inst. Henri Poincare 12 (1995), 507-575. MR 96h:35071

Department of Mathematics, East China Normal University, Shanghai 200062, PeoPLE'S RePUBlic OF CHinA

E-mail address: xnma@math.ecnu.edu.cn 